\title{
miR-190, CDK1, MCM10 and NDC80 predict the prognosis of the patients with lung cancer
}

\author{
Li-Wei Gao ${ }^{1}$, Guo-Liang Wang ${ }^{2 *}$ \\ 1. Department of Oncology, General Hospital of Pingmei Shenma Medical Group, Pingdingshan, \\ Henan 467000, China \\ 2. Department of Research \& Development, Henan Zhongping Genetic Technology Co., Ltd., \\ Zhengzhou, Henan 450000, China
}

\begin{abstract}
Lung cancer (LC), which includes small-cell lung carcinoma (SCLC) and non-small-cell lung carcinoma (NSCLC), is common and has a high fatality rate. This study aimed to reveal the prognostic mechanisms of LC. GSE30219 was extracted from the Gene Expression Omnibus (GEO) database, and included 293 LC samples and 14 normal lung samples. Differentially expressed genes (DEGs) were identified using the Limma package, and subjected to pathway enrichment analysis using DAVID. MicroRNAs (miRNAs) targeting the DEGs were predicted using Webgestalt. Cytoscape software was used to build a protein-protein interaction (PPI) network and to identify significant network modules. Survival analysis was conducted using Survminer and Survival packages, and validation was performed using The Cancer Genome Atlas (TCGA) dataset. The good and poor prognosis groups contained 518 DEGs. miR-190, miR-493, and miR-218 for the upregulated genes and miR-302, miR-200, and miR-26 for the downregulated genes were predicted. Three network modules (module 1, 2, and 3) were identified from the PPI network. CDK1, MCM10, and NDC80 were the core nodes of module 1, 2, and 3, respectively. In module 1, CDK1 interacted with both CCNB1 and CCNB2. Additionally, CDK1, CCNB1, CCNB2, MCM10, and NDC80 expression levels correlated with clinical survival and were identified as DEGs in both GSE30219 and the TCGA dataset. miR190, miR-493, miR-218, miR-200, and miR-302 might act in LC by targeting the DEGs. CDK1, CCNB1, CCNB2, $M C M 10$, and NDC80 might also influence the prognosis of LC.
\end{abstract}

Keywords: lung cancer, differentially expressed genes, microRNAs, enrichment analysis, protein-protein interaction network

Received: $3^{\text {rd }}$ April 2018; Accepted: 27 $7^{\text {st }}$ October 2018; Published: $27^{\text {th }}$ December 2018

\section{Introduction}

As a malignant lung tumor, lung cancer (LC) is mainly divided into small-cell lung carcinoma (SCLC) and non-small-cell lung carcinoma (NSCLC) (1). Patients with LC usually suffer from weight loss, coughing, chest pains, and heavy breathing (2). LC, which is the second most common tumor in women and the tumor with the highest mortality in men, affected 1.8 million people and led to 1.6 million deaths

\footnotetext{
*Corresponding author: Guo-Liang Wang, Henan Zhongping Genetic Technology Co., Ltd., No.20 Shangwuwaihuan Road, Zhengdongxin District, Zhengzhou, Henan 450000, China. E-mail: Guoliang_W800@126.com
} 
globally in 2012 (3). Therefore, the mechanisms of LC development and progression should be determined.

Peptidylprolyl cis/trans isomerase, NIMA-interacting 1 (PIN1) functions in the occurrence and metastasis of NSCLC, and thus may be a predictor for poor prognosis and a therapeutic target for the disease $(4,5)$. The oncoprotein cancerous inhibitor of protein phosphatase $2 \mathrm{~A}(C I P 2 A)$ is highly expressed in NSCLC, and is related to unfavorable prognosis and tumor cell proliferation $(6,7)$. In addition, the overexpression of Zonula Occludens-1 (ZO-1, gene symbol TJP1) contributes to the good prognosis of patients with NSCLC (8). Furthermore, upregulated expression of micro RNA (miRNA) miR-21, and downregulated expression of $m i R-143$ and $m i R-181 a$ were detected in LC tissues, indicating that these miRNAs might be potential diagnostic or prognostic factors for the patients with NSCLC (9). Although the above studies have reported some genes and miRNAs that correlated with LC, the pathogenesis of the disease remains unclear.

In 2013, Rousseaux et al. developed an approach to explore the malignant epigenetic reprogramming of LC and identified a series of candidate cancer biomarkers (10). However, they did not fully reveal the prognosis-associated genes in LC using bioinformatic analyses. In the present study, using the microarray data of Rousseaux et al. (10), the prognostic mechanisms of LC were studied through differential expression analysis, enrichment analysis, mRNA-target prediction, protein-protein interaction (PPI) network analysis, and survival analysis.

\section{Materials and Methods}

\section{Microarray data}

The gene expression profile GSE30219 (https:// www.ncbi.nlm.nih.gov/geo/query/acc.cgi?ac$\mathrm{c}=\mathrm{GSE} 30219)$ was extracted from the Gene Expression Omnibus (GEO) database, which was sequenced on the platform of GPL570 [HG-U133_Plus_2] Affymetrix Human Genome U133 Plus 2.0 Array. GSE30219 contained data for $293 \mathrm{LC}$ samples and 14 normal lung samples.

\section{Ethics approval and consent to participate}

This study was approved by Ethics Committee of the General Hospital of Pingmei Shenma Medical Group.

\section{Differential expression analysis and enrich- ment analysis}

Using the Robust Multichip Average (RMA) algorithm (11), normalization and gene expression calculations were conducted for the original data. The top $50 \mathrm{LC}$ samples with the longest or the shortest postoperative follow-up times, respectively, were classified into good and poor prognosis groups. To identify prognosis-associated genes, the differentially expressed genes (DEGs) between the good and poor prognosis groups were analyzed using the Limma (http://www.bioconductor.org/packages/release/bioc/html/limma.html) package (12) in the $\mathrm{R}$ software. The screening criteria were set as $\mid \log$ fold change (FC) $\mid>0.58$ and adjusted $\mathrm{p}$-value $<0.05$. Using the Database for Annotation, Visualization and Integrated Discovery (DAVID, https://david.ncifcrf.gov/) tool (13), the significant Kyoto Encyclopedia of Genes and Genomes (KEGG) (14) pathways involving the DEGs were identified.

\section{Prediction of miRNAs targeting the DEGs}

MiRNAs can regulate the transcription levels of target genes by binding with their 3' untranslated regions (15). Therefore, the dysregulated expression of the DEGs might also be caused by miRNA regulation. The web-based gene set analysis toolkit (Webgestalt, http://www.webgestalt.org) tool (16) was used to predict the miRNAs that target the upregulated and downregulated genes. 


\section{Protein-protein interaction network analysis}

The DEGs were submitted to the Search Tool for the Retrieval of Interacting Genes (STRING, http://string-db.org/) database (17), and the PPIs with combined scores $>0.4$ were selected. Subsequently, the PPI network was built using Cytoscape software (http://www.cytoscape.org) (18) and the clusterMaker plug-in (19) in Cytoscape was used to identify the significant network modules. The module nodes were then identified using Gene Ontology (GO) (20) functional enrichment analysis using the BinGO plug-in (21) in Cytoscape.

\section{Survival analysis}

Using the Survminer (22) and Survival (23) packages in R, survival analysis for the key genes was carried out. Based on the gene expression value, survival time, and survival state, the Survminer package could divide the samples into high expression and low expression groups. After grouping, survival analysis was conducted using the Survival package and a survival curve was drawn.

\section{Validation using an independent dataset}

The expression profile data for LC was downloaded from The Cancer Genome Atlas (TCGA) database, which included 502 LC samples and 49 normal samples. Based on the probe annotation file, the probes were mapped into gene symbols. More than one probe might correspond to the same gene symbol; therefore, their average value was calculated as the gene expression value. Differential expression analysis was then conducted using the R package, edgeR (24) (http://www.bioconductor.org/packages/ release/bioc/html/edgeR.html), with the thresholds of $|\log \mathrm{FC}|>0.58$ and adjusted $\mathrm{p}$-value $<$ 0.05. Using the VENNY tool (http://bioinfogp. cnb.csic.es/tools/venny/index.html) (25), the DEGs for GSE30219 and the TCGA dataset were compared to obtain the intersecting genes.

\section{Results}

\section{Differential expression analysis and enrich- ment analysis}

The top 50 LC samples with the longest (average follow-up time $=100.5$ months) or the shortest (average follow-up time $=5.7$ months) postoperative follow-up time were divided into good and poor prognosis groups, respectively. There were 518 DEGs (319 upregulated and 199 downregulated) in the two groups. KEGG pathway enrichment analysis suggested that the upregulated genes were significantly involved in the cell cycle. However, the downregulated genes had no significantly enriched pathways.

\section{Prediction of miRNAs targeting the DEGS}

Multiple miRNAs were predicted to target the upregulated and downregulated genes. The upregulated genes were mainly targeted by $m i R$ 190, miR-493, and miR-218 (Table 1A). Meanwhile, the downregulated genes were mainly targeted by $m i R-302, m i R-200$, and $m i R-26$ (Table 1B).

\section{PPI network analysis}

For the DEGs, a PPI network (involving 110 upregulated and 39 downregulated genes) was built. Subsequently, three network modules (module 1, 2, and 3) were obtained in which the core nodes were cyclin-dependent kinase 1 (CDK1), minichromosome maintenance 10 (MCM10), and NDC80 kinetochore complex component (NDC80), respectively (Figure 1). In module $1, \mathrm{CDK} 1$ could interact with both cyclin B1 (CCNB1) and cyclin B2 (CCNB2). GO functional enrichment analysis indicated that the module nodes were enriched in the $\mathrm{M}$ phase of the mitotic cell cycle (module $1, \mathrm{p}$-value = 4.91E-24), DNA replication (module 2, p-value $=4.44 \mathrm{E}-13)$, and $\mathrm{M}$ phase $($ module $3, \mathrm{p}$-value $=$ 1.21E-13) (Table 2). 
Table 1. The miRNAs targeting the up-regulated genes (A) and the down-regulated genes (B).

A

\begin{tabular}{|c|c|c|c|}
\hline MicroRNA & $\begin{array}{l}\text { Gene } \\
\text { count }\end{array}$ & Gene symbol & $\begin{array}{c}\text { Adjusted } \\
\text { p-value }\end{array}$ \\
\hline hsa_ACATATC,MIR-190 & 6 & CELF4,CHD7,ANO4,PHLPP1,STC1,NEUROD1 & 0.0007 \\
\hline hsa_ATGTACA,MIR-493 & 11 & $\begin{array}{l}\text { ZNF804A,ZNF711,ATAD2,SALL1,NCAPH,- } \\
\text { JPH1,POU4F1,ANO4,CDH2,GAD1,MAP1B }\end{array}$ & 0.0013 \\
\hline hsa_AAGCACA,MIR-218 & 12 & $\begin{array}{l}\text { TRIM9,RIMBP2,PCDH8,LMNB1,INHBB,ST18,PEX5L,PH- } \\
\text { F16,SATB2,ANO4,FANCI,HOXD10 }\end{array}$ & 0.0013 \\
\hline hsa_CATGTAA,MIR-496 & 8 & $\begin{array}{l}\text { CDKN2C,CHD7,DPP10,HMP19,ASCL1,NEUROD1,KCNH8,- } \\
\text { SATB2 }\end{array}$ & 0.0013 \\
\hline hsa_TAGCTTT,MIR-9 & 9 & $\begin{array}{l}\text { POU3F2,ELAVL2,CKAP2L,INSM1,TARDBP,PH- } \\
\text { F16,POU4F1,HMGB2,STC1 }\end{array}$ & 0.0016 \\
\hline $\begin{array}{l}\text { hsa_TGTTTAC,MIR-30A- } \\
\text { 5P,MIR-30C,MIR-30D,MIR- } \\
\text { 30B,MIR-30E-5P }\end{array}$ & 14 & $\begin{array}{l}\text { RIMBP2,CCNE2,CHD7,NEUROD1,PHF16,SAT- } \\
\text { B2,NKX2-2,ANO4,SCN2A,FOXG1,TTLL7,STC1,SC- } \\
\text { N3A,RHEBL1 }\end{array}$ & 0.0018 \\
\hline $\begin{array}{l}\text { hsa_TTTGCAC,MIR-19A,MIR- } \\
\text { 19B }\end{array}$ & 13 & $\begin{array}{l}\text { DPYSL5,ZNF711,POU3F2,ZBTB10,INHBB,NEURO- } \\
\text { D1,PRC1,CBLN2,PEX5L,POU4F1,SYT1,E2F8,RHEBL1 }\end{array}$ & 0.0018 \\
\hline hsa_CTATGCA,MIR-153 & 8 & $\begin{array}{l}\text { DPYSL5,INHBB,NEUROD1,POU4F1,DLX1,C9orf40,SYT1,- } \\
\text { TAGLN3 }\end{array}$ & 0.0023 \\
\hline hsa_ACTGCCT,MIR-34B & 8 & $\begin{array}{l}\text { RIMBP2,ICA1,WASF1,NEUROD1,ST18,POU4F1,INS- } \\
\text { M1,STC1 }\end{array}$ & 0.0023 \\
\hline hsa_ATGCAGT,MIR-217 & 6 & ZNF711,STX1A,NEUROD1,ST18,RBM38,EZH2 & 0.0023 \\
\hline
\end{tabular}

B

\begin{tabular}{|c|c|c|c|}
\hline MicroRNA & $\begin{array}{l}\text { Gene } \\
\text { count }\end{array}$ & Gene symbol & $\begin{array}{c}\text { Adjusted } \\
\text { p-value }\end{array}$ \\
\hline $\begin{array}{l}\text { hsa_AGCACTT,MIR-93,MIR-302A,MIR- } \\
302 \text { B, MIR - } 302 \text { C, M I R - } 302 \text { D, MIR - } \\
\text { 372,MIR-373,MIR-520E,MIR-520A,MIR- } \\
\text { 526B,MIR-520B,MIR-520C,MIR-520D }\end{array}$ & 8 & $\begin{array}{l}\text { HLF,TBC1D8B,RAB11FIP1,ADAM9,NTN4,CCND2,S- } \\
\text { DC1,MTUS1 }\end{array}$ & 0.0129 \\
\hline 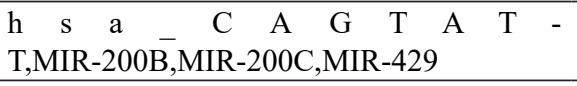 & 9 & $\begin{array}{l}\text { TRIM2,HLF,GLI3,KCNJ2,FGFR2,DGKA,ZNF217,CIT- } \\
\text { ED2,MTUS1 }\end{array}$ & 0.0129 \\
\hline hsa_TACTTGA,MIR-26A,MIR-26B & 7 & PTGS2,KCNJ2,GRHL3,HPGD,NTN4,ZNF217,CCND2 & 0.0143 \\
\hline $\begin{array}{l}\text { hsa_TGGTGCT,MIR-29A,MIR-29B,MIR- } \\
\text { 29C }\end{array}$ & 9 & $\begin{array}{c}\text { CLDN1,MUC4,ZFP36,RNF39,CCND2,SH- } \\
\text { ROOM2,AHR,CAV2,IL1RAP } \\
\end{array}$ & 0.0150 \\
\hline hsa_CATTTCA,MIR-203 & 6 & GLI3,ELL2,GRHL3,EDN1,CITED2,AHR & 0.0344 \\
\hline hsa_TTTGCAC,MIR-19A,MIR-19B & 8 & LBH,HLF,ELL2,KCNJ2,ZNF217,CCND2,SDC1,IGFBP3 & 0.0373 \\
\hline hsa_AACTGGA,MIR-145 & 5 & TRIM2,MPZL2,NEDD9,CITED2,STEAP4 & 0.0528 \\
\hline $\begin{array}{l}\text { h s a _G C C A C T T T, M I R - } 17 \text { - } 5 \text { P, M I R - } \\
\text { 20A,MIR-106A,MIR-106B,MIR-20B,MIR- } \\
\text { 519D }\end{array}$ & 8 & $\begin{array}{l}\text { HLF,TBC1D8B,SGMS1,RAB11FIP1,ADAM9,NT- } \\
\text { N4,ZNF217,CCND2 }\end{array}$ & 0.0554 \\
\hline hsa_ACTGTGA,MIR-27A,MIR-27B & 7 & $\begin{array}{l}\text { LBH,ELL2,SGMS1,LPAR6,RAB11FIP1,CACNA2D3,G- } \\
\text { PR126 }\end{array}$ & 0.0554 \\
\hline hsa_AGCTCCT,MIR-28 & 3 & LBH,NDRG2,SDC1 & 0.0800 \\
\hline
\end{tabular}




\section{Survival analysis}

After the samples in GSE30219 were divided into high expression and low expression groups, survival analysis for $C D K 1, C C N B 1, C C N B 2$, $M C M 10$, and $N D C 80$ was performed. The survival curves showed that the expression in all five genes correlated with the survival of patients with LC (p-value < 0.0001) (Figure 2).

\section{Validation using an independent dataset}

For the TCGA dataset, a total of 15226 DEGs were obtained. After comparing the DEGs for GSE30219 and the TCGA dataset, 412 intersecting genes (including $C D K 1, C C N B 1, C C N B 2$, $M C M 10$, and $N D C 80$ ) were identified (Figure 3).

\section{Discussion}

In this study, 518 DEGs in the good and poor prognosis groups were screened. $m i R-190, m i R-$
493 , and $m i R-218$ were the main miRNAs predicted to target the upregulated genes. Meanwhile, $m i R-302$, $m i R-200$, and $m i R-26$ were predicted to target the downregulated genes. From the PPI network for the proteins encoded by the DEGs, three network modules (module 1, 2, and 3) were identified. CDK1, MCM10, and NDC 80 were the core nodes of module 1,2 , and 3 , respectively. Survival analysis showed that $C D K 1$, $C C N B 1, C C N B 2, M C M 10$, and NDC80 expression levels correlated with the survival of LC patients. In addition, 412 intersecting genes (including $C D K 1, C C N B 1, C C N B 2, M C M 10$, and $N D C 80$ ) were identified between the DEGs for GSE30219 and the TCGA dataset.

Increased $m i R-190$ expression results in the low protein levels of forkhead box P2 (FOXP2); thus, miR-190 may be a promising diagnostic
A

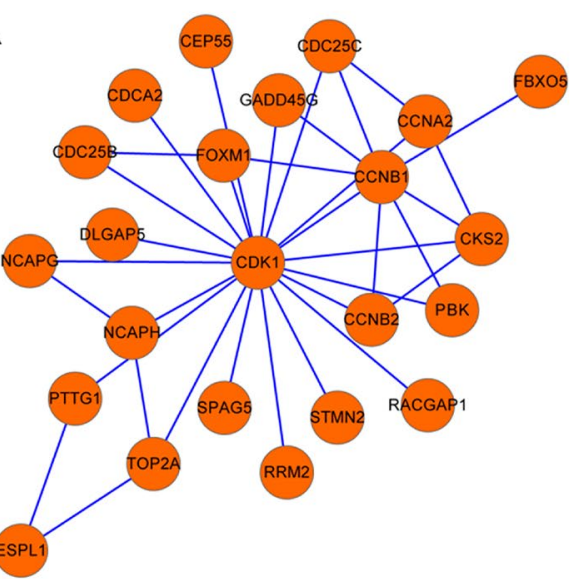

B

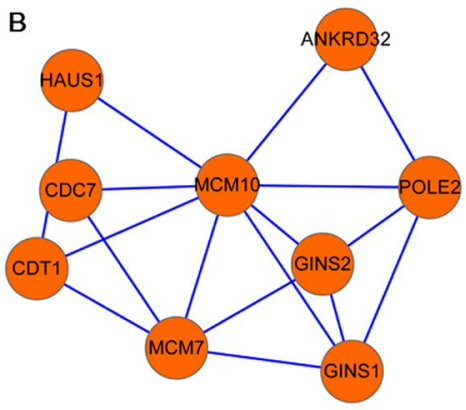

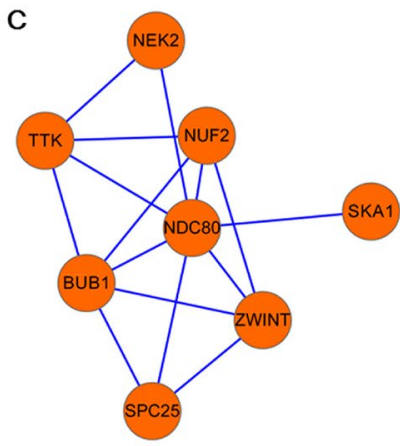

Fig. 1. Modules 1 (A), 2 (B), and 3 (C) obtained from protein-protein interaction (PPI) network.

Table 2. The Gene Ontology (GO) terms enriched for the nodes in module 1, 2 and 3.

\begin{tabular}{lcccc}
\hline Module & Term & Count & P-value & Genes \\
\hline Module 1 & $\begin{array}{c}\text { M phase of } \\
\text { mitotic cell } \\
\text { cycle }\end{array}$ & 16 & $4.91 \mathrm{E}-24$ & $\begin{array}{c}\text { CDK1, DLGAP5, ESPL1, CEP55, PBK, PTTG1, CDC25C, CDC25B, } \\
\text { CCNB1, NCAPH, CCNB2, NCAPG, SPAG5, CDCA2, FBXO5, } \\
\text { CCNA2 }\end{array}$ \\
\hline Module 2 & $\begin{array}{c}\text { DNA replica- } \\
\text { tion }\end{array}$ & 7 & $4.44 \mathrm{E}-13$ & CDC7, GINS1, GINS2, MCM7, POLE2, MCM10, CDT1 \\
\hline Module 3 & M phase & 8 & $1.21 \mathrm{E}-13$ & SPC25, NEK2, ZWINT, BUB1, NUF2, TTK, NDC80, SKA1 \\
\hline
\end{tabular}



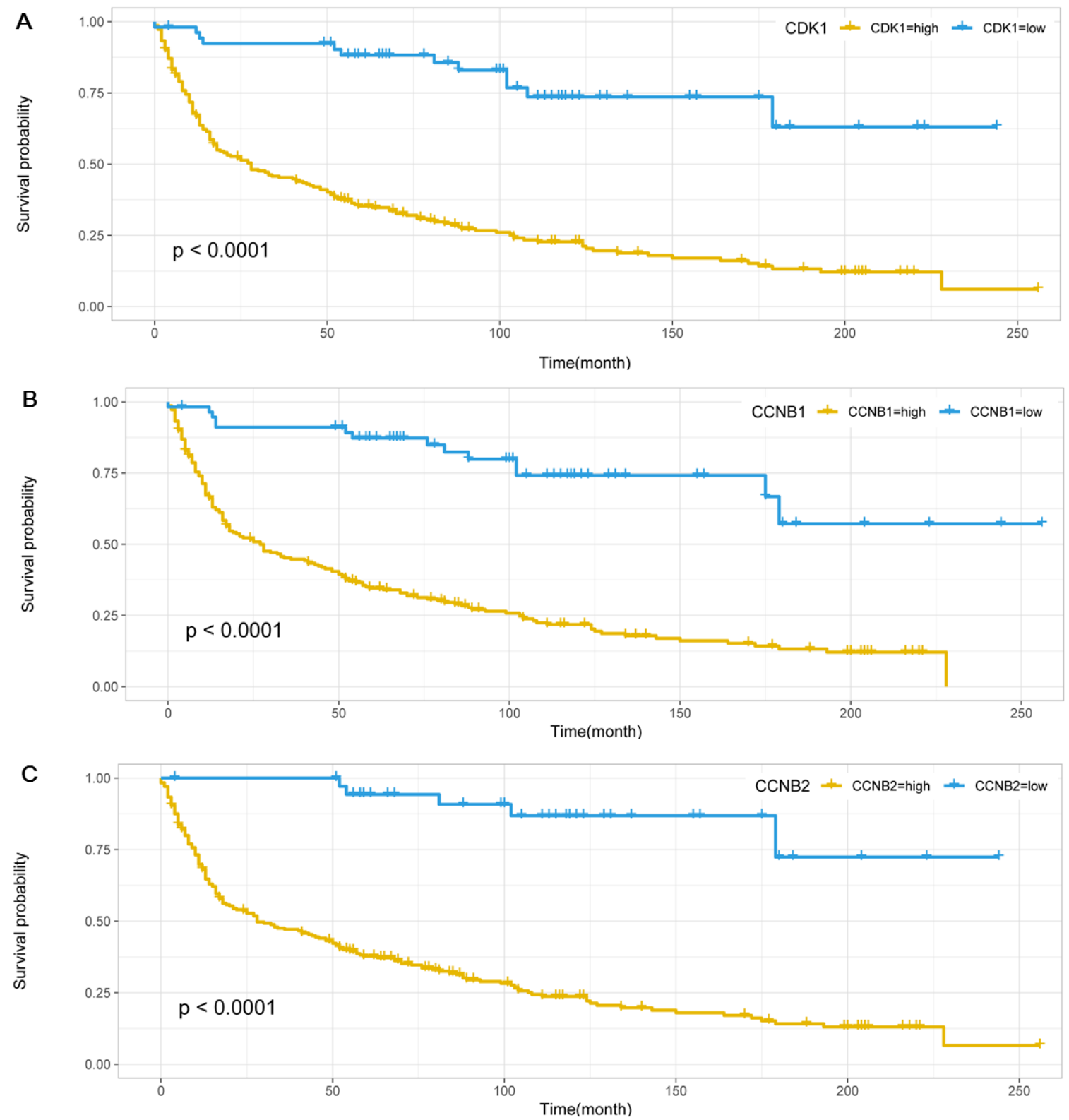

Fig. 2A-C. The survival curves for CDK1 (cyclin-dependent kinase 1) (A), CCNB1 (cyclin B1) (B), and CCNB2 (cyclin B2) (C). Yellow and blue represent the high and low expression groups, respectively.

marker for gastric cancer (26). Downregulated expression of $m i R-190$ inhibits the metastasis of breast cancer via inversely regulating the expression of SMAD family member 2 (SMAD2), which is correlated with the prog- nosis of patients with breast cancer (27). By targeting integrin, beta 1 (ITGB1), miR-493-5p plays a prognostic role in NSCLC and could be used to diagnose the disease (28). Four upregulated miRNAs and two downregulated miR- 

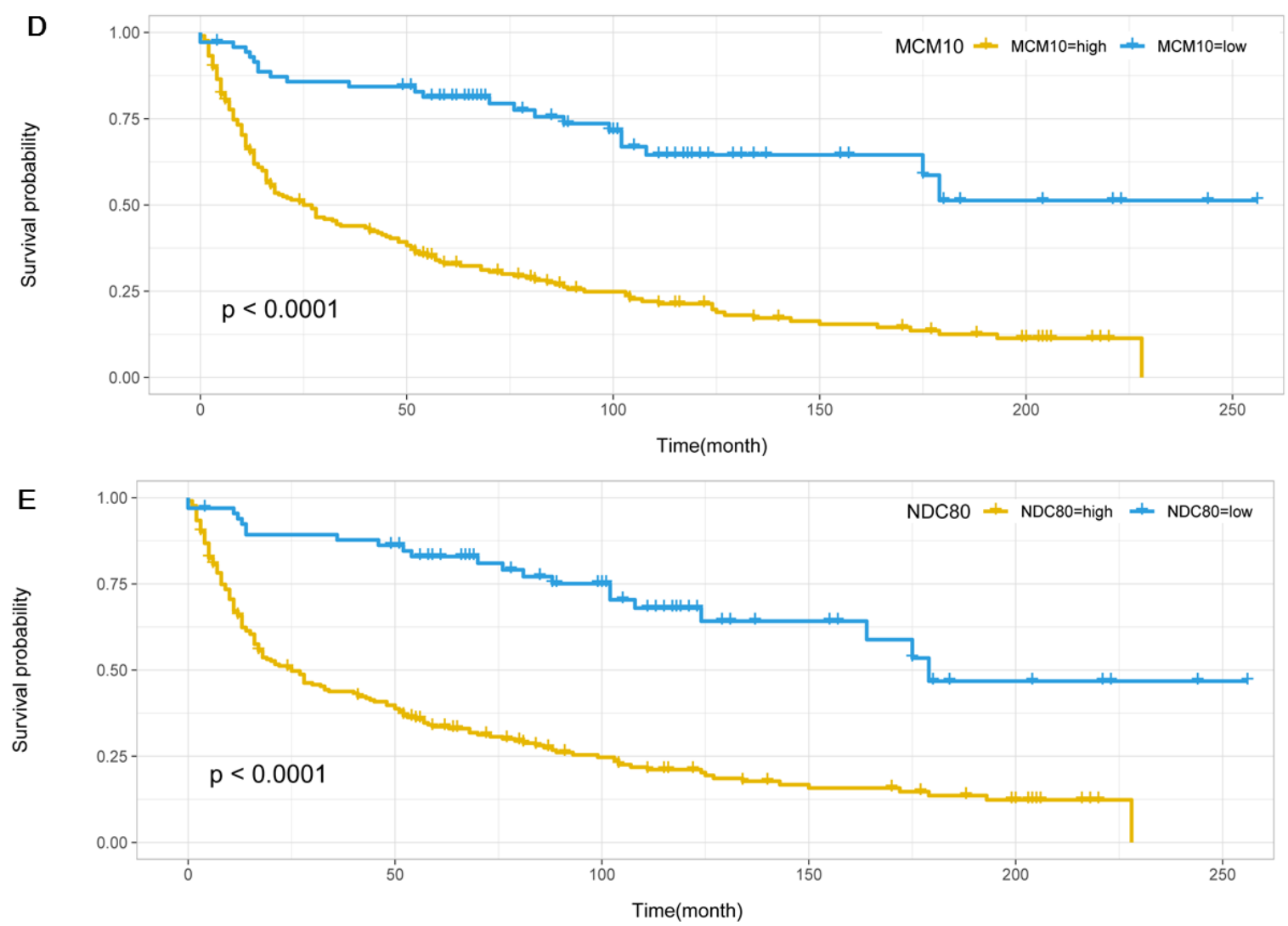

Fig. 2D-E. The survival curves for MCM10 (minichromosome maintenance 10) (D), and NDC80 (NDC80 kinetochore complex component) (E). Yellow and blue represent the high and low expression groups, respectively.

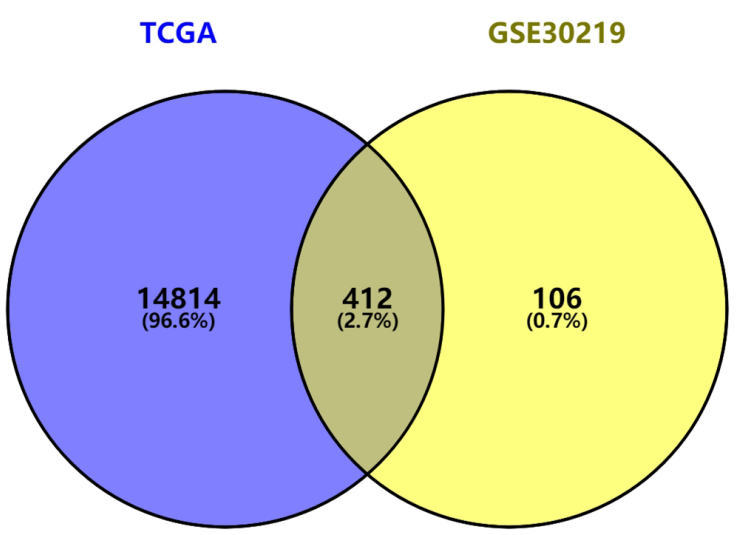

Fig. 3. Venn diagram used to identify the intersecting genes between the differentially expressed genes for GSE30219 and The Cancer Genome Atlas (TCGA) dataset.
NAs (including $m i R-218-5 p$ ) were identified in lung adenocarcinoma (LUAD) tissues and may represent promising diagnostic and prognostic markers for patients with LUAD (29). Overexpression of $m i R-200 c$ plays an oncogenic role in NSCLC and correlates markedly with adverse prognosis; thus, it may serve as a prognostic biomarker for patients with NSCLC (30). Exosome-derived miR-302b mediates the transforming growth factor beta (TGF beta RII)/ extracellular signal-regulated kinase (ERK) pathway, which can inhibit cell proliferation and migration in LC (31). Therefore, $m i R$ 190, miR-493, miR-218, miR-200, and miR-302 might affect the prognosis of LC by regulating the expression of the DEGs. 
$C D K 1$ and MAD2 mitotic arrest deficient-like 1 (MAD2L1) can lead to high risk of recurrence and poor prognosis of patients with LUAD; therefore, they are negatively correlated with prognosis and may be promising therapeutic targets for the disease (32). CCNB1 and CDK1 levels are higher in LC compared with those in normal tissues, and their expression levels vary among different types of LC $(33,34)$. CCNB1 and cyclin A $(C C N A)$ have dysregulated expression in NSCLC, and CCNB1 may serve as a potential prognostic factor for patients with NSCLC (35). CCNB2 is overexpressed in LUAD cells, which independently predicts poor survival of patients with LUAD (36). CDK1 was predicted to interact with both CCNB1 and CCNB2 in module 1, suggesting that CDK1 might affect the prognosis of LC via interactions with CCNB1 and CCNB2.

Minichromosome maintenance (MCM) proteins are involved in multiple cancers. In particular, MCM5 and MCM7 are independent prognostic markers for patients with LC $(37,38)$. MCM4 is highly expressed in NSCLC and is related to non-adenocarcinoma histology, indicating that it might represent a therapeutic target in NSCLC (39). MCM2, MCM3, MCM7, MCM10, polo-like kinase 1 ( $P L K 1)$, and S-phase kinase associated protein 2 (SKP2) are involved in cancer-associated pathways and may coordinately facilitate cell cycle-associated processes in tumors (40). Cell division associated 1 (CDCA1) and NDC80 (also called KNTC2) are simultaneously upregulated in LC, which is a critical feature of tumor cell growth and survival and provides a valuable therapeutic strategy for the disease (41). Therefore, our results, and those of previous studies, demonstrated that MCM10 and NDC80 might also be related to LC prognosis.

Although multiple bioinformatic methods were used to explore the prognostic mechanisms of LC, this study had several limitations. The lack of experimental validation was the most prominent limitation. Therefore, further experimental research is needed to support these results.

In conclusion, 518 DEGs were screened from the good and poor prognosis groups. MicroRNAs $m i R-190, m i R-493, m i R-218, m i R-200$, and $m i R-302$ might correlate with the prognosis of $\mathrm{LC}$ by regulating the expression levels of certain DEGs. Furthermore, $C D K 1, C C N B 1, C C N B 2$, $M C M 10$, and NDC80 might be associated with the prognosis of LC.
Abbreviations
Lung cancer (LC)
Small-cell lung carcinoma (SCLC)
Non-small-cell lung carcinoma (NSCLC)
Gene Expression Omnibus (GEO)
Differentially expressed genes (DEGs)
Protein-protein interaction (PPI)
Robust Multichip Average (RMA)
Gene Ontology (GO)
Kyoto Encyclopedia of Genes and Genomes (KEGG)
Cyclin-dependent kinase 1 (CDK1)
Minichromosome maintenance 10 (MCM10)
NDC80 kinetochore complex component
(NDC80)
Cyclin B1 (CCNB1)
Cyclin B2 (CCNB2)

\section{Conflict of interst}

The authors declare that they have no potential conflict of interest. This study was not supported by any external funding

\section{Authors' contributions}

L-WG conceived and designed the research, participated in the acquisition of data, and drafted the manuscript.

G-LW carried out the analysis and interpretation of data, and participated in the statistical analysis. L-WG and G-LW conceived the study, partici- 
pated in its design and coordination, and helped to draft the manuscript. All authors read and approved the final manuscript.

\section{References}

1. Travis WD, Travis LB, Devesa SS. Lung cancer. Cancer. 2015;75(S1):191-202. DOI: 10.1002/1097-0142(19950101)75:1+<191::AID-CNCR2820751307>3.0.CO;2-Y

2. Collins LG, Haines C, Perkel R, Enck RE. Lung cancer: diagnosis and management. Am Fam Physician. 2007;75(1):56-63.

3. Mcguire S. World Cancer Report 2014. Geneva, Switzerland: World Health Organization, International Agency for Research on Cancer, WHO Press, 2015. Adv Nutr. 2016;7(2):418. DOI: 10.3945/an.116.012211

4. Tan X, Fang Z, Wan J, Jie H, Chen Z, Li B et al. Pin1 expression contributes to lung cancer prognosis and carcinogenesis. Cancer Biol Ther. 2010;9(2):111-9. DOI: $10.4161 /$ cbt.9.2.10341

5. Yoon HE, Kim SA, Choi HS, Ahn MY, Yoon JH, Ahn SG. Inhibition of Plk1 and Pin1 by 5 -nitro-indirubinoxime suppresses human lung cancer cells. Cancer Lett. 2012;316(1):97-104. DOI: 10.1016/j.canlet.2011.10.029

6. Dong QZ, Wang Y, Dong XJ, Li ZX, Tang ZP, Cui QZ et al. CIP2A is Overexpressed in Non-Small Cell Lung Cancer and Correlates with Poor Prognosis. Ann Surg Oncol. 2011;18(3):857. DOI: 10.1245/s10434-0101313-8

7. Xu P, Xu XL, Huang Q, Zhang ZH, Zhang YB. CIP2A with survivin protein expressions in human non-small. Med Oncol. 2012;29(3):1643-7. DOI: 10.1007/s12032011-0053-3

8. Ni S, Xu L, Huang J, Feng J, Zhu H, Wang G et al. Increased ZO-1 expression predicts valuable prognosis in non-small cell lung cancer. Int J Clin Exp Pathol. 2013;6(12):2887-95.

9. Gao W, Yu Y, Cao H, Shen H, Li X, Pan S et al. Deregulated expression of miR-21, miR-143 and miR-181a in non small cell lung cancer is related to clinicopathologic characteristics or patient prognosis. Biomed Pharmacother. 2010;64(6):399. DOI: 10.1016/j.biopha.2010.01.018

10. Rousseaux S, Debernardi A, Jacquiau B, Vitte AL, Vesin A, Nagymignotte $\mathrm{H}$ et al. Ectopic Activation of Germline and Placental Genes Identifies Aggressive Metastasis-Prone Lung Cancers. Sci Transl Med. 2013;5(186):186ra66. DOI: 10.1126/scitranslmed.3005723

11. Irizarry RA, Wu Z, Jaffee HA. Comparison of Affymetrix GeneChip expression measures. Bioinformatics. 2006;22(7):789. DOI: 10.1093/bioinformatics/btk046
12. Ritchie ME, Phipson B, Wu D, Hu Y, Law CW, Shi W et al. limma powers differential expression analyses for RNA-sequencing and microarray studies. Nucleic Acids Res. 2015;43(7). DOI: 10.1093/nar/gkv007

13. Huang DW, Sherman BT, Lempicki RA. Systematic and integrative analysis of large gene lists using DAVID bioinformatics resources. Nat Protoc. 2009;4(1):44. DOI: $10.1038 /$ nprot.2008.211

14. Kanehisa M, Sato Y, Kawashima M, Furumichi M, Tanabe M. KEGG as a reference resource for gene and protein annotation. Nucleic Acids Res. 2015;44(D1):D457-D62. DOI: 10.1093/nar/gkv1070

15. He L, Hannon GJ. MicroRNAs: small RNAs with a big role in gene regulation. Nat Rev Genet. 2004;5(7):52231. DOI: $10.1038 / \operatorname{nrg} 1379$

16. Wang J, Duncan D, Shi Z, Zhang B. WEB-based GEne SeT AnaLysis Toolkit (WebGestalt): update 2013. Nucleic Acids Res. 2013;41(W1):77-83. DOI: 10.1093/ nar/gkt439

17. Franceschini A, Szklarczyk D, Frankild S, Kuhn M, Simonovic M, Roth A et al. STRING v9. 1: protein-protein interaction networks, with increased coverage and integration. Nucleic Acids Res. 2013;41(D1):D808-D15. DOI: 10.1093/nar/gks1094

18. Saito R, Smoot ME, Ono K, Ruscheinski J, Wang P-L, Lotia $\mathrm{S}$ et al. A travel guide to Cytoscape plugins. Nat Methods. 2012;9(11):1069-76. DOI: 10.1038/ nmeth. 2212

19. Morris JH, Apeltsin L, Newman AM, Baumbach J, Wittkop T, Su G et al. clusterMaker: a multi-algorithm clustering plugin for Cytoscape. BMC bioinformatics. 2011;12:436 DOI: 10.1186/1471-2105-12-436. DOI: $10.1186 / 1471-2105-12-436$

20. Consortium TGO. Gene Ontology Consortium: going forward. Nucleic Acids Res. 2015;43(Database issue):1049-56. DOI: 10.1093/nar/gku1179

21. Maere S, Heymans K, Kuiper M. BiNGO: a Cytoscape plugin to assess overrepresentation of gene ontology categories in biological networks. Bioinformatics. 2005 Aug 15;21(16):3448-9 DOI: 10.1093/bioinformatics/ bti551. DOI: 10.1093/bioinformatics/bti551

22. Kassambara A. survminer: Drawing Survival Curves using 'ggplot2'. R package version 0.2.2. ed. https:// CRAN.R-project.org/package=survminer. 2016.

23. Therneau TM, April. A Package for Survival Analysis in S. version 2.38 ed. http://CRAN.R-project.org/package $=$ survival. 2015 .

24. Robinson MD, Mccarthy DJ, Smyth GK. edgeR: a Bioconductor package for differential expression analysis of digital gene expression data. Bioinformatics. 2010;26(1):139. DOI: 10.1093/bioinformatics/btp616

25. Oliveros JC. VENNY. An interactive tool for comparing lists with Venn Diagrams. http://bioinfogp.cnb.csic. es/tools/venny/index.html. 2007.

26. Jia WZ, Tao Y, Qi A, Hua Y, Zhu Z, Xiao L et al. Mi- 
croRNA-190 regulatesFOXP2genes in human gastric cancer. Onco Targets Ther. 2016;9(Issue 1):3643-51.

27. Yu Y, Luo W, Yang ZJ, Chi JR, Li YR, Ding Y et al. miR-190 suppresses breast cancer metastasis by regulation of TGF- $\hat{I}^{2}$-induced epithelial-mesenchymal transition. Mol Cancer. 2018;17(1):70. DOI: 10.1186/ s12943-018-0818-9

28. Liang Z, Kong R, He Z, Lin LY, Qin SS, Chen CY et al. High expression of miR-493-5p positively correlates with clinical prognosis of non small cell lung cancer by targeting oncogene ITGB1. Oncotarget. 2017;8(29):47389-99. DOI: 10.18632/oncotarget. 17650

29. Peng Z, Pan L, Niu Z, Li W, Dang X, Lin W et al. Identification of microRNAs as potential biomarkers for lung adenocarcinoma using integrating genomics analysis. Oncotarget. 2017;8(38):64143. DOI: 10.18632/ oncotarget. 19358

30. Si L, Tian H, Yue W, Li L, Li S, Gao C et al. Potential use of microRNA-200c as a prognostic marker in nonsmall cell lung cancer. Oncol Lett. 2017;14(4):4325. DOI: $10.3892 / \mathrm{ol} .2017 .6667$

31. Li J, Yu J, Zhang H, Wang B, Guo H, Bai J et al. Exosomes-Derived MiR-302b Suppresses Lung Cancer Cell Proliferation and Migration via TGF beta RII Inhibition. Cell Physiol Biochem. 2016;38(5):1715. DOI: 10.1159/000443111

32. Shi YX, Zhu T, Zou T, Zhuo W, Chen YX, Huang MS et al. Prognostic and predictive values of CDK1 and MAD2L1 in lung adenocarcinoma. Oncotarget. 2016;7(51):85235. DOI: 10.18632/oncotarget.13252

33. Huang SH, Xiao-Li MA, Qiu C, Huang JA, Kong WH, Xie JW et al. The overexpression of cyclin B1 and CDK1 in lung carcinoma and its clinical significance. Journal of Shandong University. 2004;39(5):122-4.

34. Jacquot C, Rousseau B, Carbonnelle D, Chinou I, Malleter $\mathrm{M}$, Tomasoni $\mathrm{C}$ et al. Cucurbitacin-D-induced
CDK1 mRNA up-regulation causes proliferation arrest of a non-small cell lung carcinoma cell line (NSCLC-N6). Anticancer Res. 2014;34(9):4797-806.

35. Cooper WA, Kohonencorish MR, Mccaughan B, Kennedy C, Sutherland RL, Lee CS. Expression and prognostic significance of cyclin B1 and cyclin A in nonsmall cell lung cancer. Histopathology. 2009;55(1):2836. DOI: 10.1111/j.1365-2559.2009.03331.x

36. Takashima S, Saito H, Takahashi N, Imai K, Kudo S, Atari $\mathrm{M}$ et al. Strong expression of cyclin B2 mRNA correlates with a poor prognosis in patients with nonsmall cell lung cancer. Tumour Biol. 2014;35(5):425765. DOI: $10.1007 / \mathrm{s} 13277-013-1556-7$

37. Liu YZ, Wang BS, Jiang YY, Cao J, Hao JJ, Zhang Y et al. MCMs expression in lung cancer: implication of prognostic significance. J Cancer. 2017;8(18):3641-7. DOI: $10.7150 /$ jca.20777

38. Liu YZ, Jiang YY, Hao JJ, Lu SS, Zhang TT, Shang L et al. Prognostic significance of MCM7 expression in the bronchial brushings of patients with non-small cell lung cancer (NSCLC). Lung Cancer. 2012;77(1):176. DOI: 10.1016/j.lungcan.2012.03.001

39. Kikuchia J, Kinoshitab I, Shimizub Y, Kikuchia E, Takedab K, Abu H. Minichromosome maintenance $(\mathrm{MCM})$ protein 4 as a marker for proliferation and its clinical and clinicopathological significance in nonsmall cell lung cancer. Lung Cancer. 2011;72(2):22937. DOI: 10.1016/j.lungcan.2010.08.020

40. Chao W. Integrating gene expression and protein-protein interaction network to prioritize cancer-associated genes. BMC bioinformatics. 2012;13(1):182. DOI: 10.1186/1471-2105-13-182

41. Hayama S, Daigo Y, Kato T, Ishikawa N, Yamabuki T, Miyamoto $\mathrm{M}$ et al. Activation of CDCA1-KNTC2, Members of Centromere Protein Complex, Involved in Pulmonary Carcinogenesis. 2006;66(21):10339-48. 